\title{
Kolmogorov-Chentsov Theorem and Differentiability of Random Fields on Manifolds
}

\section{Journal Article}

\section{Author(s):}

Andreev, Roman; Lang, Annika

Publication date:

2014-10

Permanent link:

https://doi.org/10.3929/ethz-b-000091461

Rights / license:

In Copyright - Non-Commercial Use Permitted

Originally published in:

Potential Analysis 41(3), https://doi.org/10.1007/s11118-014-9392-y 


\title{
Kolmogorov-Chentsov Theorem and Differentiability of Random Fields on Manifolds
}

\author{
R. Andreev • A. Lang
}

Received: 1 November 2013 / Accepted: 16 January 2014 / Published online: 3 February 2014

(C) Springer Science+Business Media Dordrecht 2014

\begin{abstract}
A version of the Kolmogorov-Chentsov theorem on sample differentiability and Hölder continuity of random fields on domains of cone type is proved, and the result is generalized to manifolds.
\end{abstract}

Keywords Random fields on manifolds · Kolmogorov-Chentsov theorem · Sample Hölder continuity $\cdot$ Sample differentiability $\cdot$ Sobolev embeddings

Mathematics Subject Classifications (2010) 60G60 • 60G17 · 46E35

\section{Introduction}

Sample regularity of random fields and stochastic processes has been first studied by Kolmogorov in the 1930's, as reported in [24], and extended by Chentsov in [6]. The Kolmogorov-Chentsov theorem states the existence of continuous modifications of stochastic processes and derives bounds on the exponent of sample Hölder continuity. This result has been extended in many ways, for example: for random fields on cubes [2], for random fields on the sphere [13], for random fields on metric spaces [10, 21], and for random fields with values in more general spaces $[17,19]$. For a review on the literature and the history of the problem we refer the reader to the introduction of [21].

R. Andreev · A. Lang

Seminar für Angewandte Mathematik, ETH Zürich, Rämistrasse 101, CH-8092 Zürich, Switzerland

R. Andreev

RICAM, Austrian Academy of Sciences, Altenberger Str. 69, A-4040 Linz, Austria

e-mail: roman.andreev@oeaw.ac.at

A. Lang $(\bowtie)$

Department of Mathematical Sciences, Chalmers University of Technology and University of Gothenburg, SE-412 96 Göteborg, Sweden

e-mail: annika.lang@chalmers.se 
The first objective of this paper is to extend these results to random fields on domains. In fact, we also show sample differentiability under suitable further assumptions on the random fields. The second objective is to extend these results to random fields on manifolds.

In contrast to Hölder continuity of stochastic processes and random fields, sample differentiability has hardly been studied so far, partly because the Brownian motion and the related stochastic processes are almost surely nowhere differentiable. In recent years, however, the question of smoothness of random fields (beyond Hölder continuity) has become more important. In particular, higher spatial smoothness of solutions of stochastic partial differential equations can be exploited to improve the order of convergence of numerical algorithms. Other examples include solutions of random partial differential equations as presented in [5], or lognormal random fields on the sphere in the modeling of ice crystals (cf. [13, 18]). Results on existence of sample differentiable modifications for stochastic processes are presented in [7] and [16]. Furthermore, differentiability on $\mathbb{R}^{n}$ of Gaussian random fields is discussed in [3] and first order sample derivatives are obtained in [22] with a differential and integral calculus in quadratic mean. As a first approach to manifolds, existence of higher order derivatives of isotropic Gaussian random fields on the sphere is shown in relation with the decay of the corresponding angular power spectrum in [13]. To the best of our knowledge, this paper is the first to provide an extension of the KolmogorovChentsov theorem to show sample differentiability of random fields on domains of cone type as well as on manifolds.

The main technical device of our proof is the Sobolev embedding theorem, as was sketched in [8, Proof of Theorem 3.4]. In general, it provides a modification (in space) of the function in question, and one would need to show that the resulting random field is a modification of the original one. We circumvent this complication by showing existence of a sample continuous modification first. Our results are stated in terms of spaces of continuously differentiable functions of fractional order, which characterize the order of differentiability and the "remaining" Hölder continuity of the highest order derivatives.

The paper is organized as follows. In Section 2 we introduce the necessary basics on Sobolev spaces, Sobolev embeddings, manifolds, and random fields, as well as our notation. Section 3 contains our two main results, which state the existence of Hölder continuous and differentiable modifications of random fields on domains of cone type, and on sufficiently smooth manifolds. The proofs can be found in the same section.

\section{Preliminaries}

In this preparatory section we collect the notions required to obtain our main results in Section 3 in the order that is needed later on. Therefore, we start with the introduction of Sobolev spaces on domains, and recall the appropriate variant of the Sobolev embedding theorem. We move on to define manifolds, and spaces of Hölder continuous and differentiable functions on them. Finally, we introduce random fields on manifolds and associated properties.

We briefly recall the theory of (fractional) Sobolev spaces and spaces of Hölder continuous and differentiable functions on domains. For details, we refer the reader to the standard literature $[1,9,25]$.

For any $t>0$, the integer part $\lfloor t\rfloor \in \mathbb{N}_{0}$ and the fractional part $\{t\} \in[0,1)$ of $t$ are uniquely determined by $t=\lfloor t\rfloor+\{t\}$. For a multi-index $\alpha \in \mathbb{N}_{0}^{n}$ we set $|\alpha|:=\sum_{i=1}^{n} \alpha_{i}$. 
A subset $D \subset \mathbb{R}^{n}$ is called a domain if it is nonempty, open, and connected. If $D$ is a domain, we define for $t \in \mathbb{N}_{0}$

$$
\|f\|_{\bar{C}^{t}(D)}:=\sum_{|\alpha| \leq t} \sup _{x \in D}\left|\partial^{\alpha} f(x)\right|,
$$

where $\partial^{\alpha}:=\partial^{|\alpha|} /\left(\partial x_{1}^{\alpha_{1}} \cdots \partial x_{n}^{\alpha_{n}}\right)$ is the classical partial derivative, and for noninteger $t>0$

$$
\|f\|_{\bar{C}^{t}(D)}:=\|f\|_{\bar{C}^{\lfloor t\rfloor}(D)}+\sum_{|\alpha| \leq\lfloor t\rfloor} \sup _{\substack{x, y \in D \\ x \neq y}} \frac{\left|\partial^{\alpha} f(x)-\partial^{\alpha} f(y)\right|}{|x-y|^{\{t\}}} .
$$

For $t>0$ we define the Hölder spaces

$$
\bar{C}^{t}(D):=\left\{f: D \rightarrow \mathbb{R} ; f \text { is }\lfloor t\rfloor \text { times continuously differentiable and }\|f\|_{\bar{C}^{t}(D)}<\infty\right\} .
$$

The Lebesgue space $L^{p}(D), p \in[1, \infty)$, comprises all measurable functions $u: D \rightarrow \mathbb{R}$ for which $\|u\|_{L^{p}(D)}^{p}:=\int_{D}|u(x)|^{p} d x$ is finite. Functions that are equal for almost every $x \in D$ are identified. For $p \in[1, \infty)$ and $k \in \mathbb{N}$, the Sobolev space $W_{p}^{k}(D)$ is defined by

$$
W_{p}^{k}(D):=\left\{u \in L^{p}(D): \partial^{\alpha} u \in L^{p}(D) \text { for all } 0 \leq|\alpha| \leq k\right\},
$$

where $\partial^{\alpha}$ denotes the distributional partial derivative. Equipped with the norm $\|\cdot\|_{W_{p}^{k}(D)}$ given by

$$
\|u\|_{W_{p}^{k}(D)}^{p}:=\sum_{0 \leq|\alpha| \leq k}\left\|\partial^{\alpha} u\right\|_{L^{p}(D)}^{p},
$$

it becomes a Banach space (see e.g., [1, Theorem 3.3]).

Finally, we extend the definition of Sobolev spaces to nonintegers for bounded domains $D$ of cone type following [25, Definition 4.2.3]. As noted there, examples of bounded domains of cone type include open cubes and bounded domains with a smooth (or $C^{1}$ ) boundary.

Definition 2.1 A bounded domain $D$ is said to be of cone type if there exist domains $U_{1}, \ldots, U_{m}$, and cones $C_{1}, \ldots, C_{m}$, which may be carried over by rotations into the cone of height $h$

$$
K_{h}:=\left\{x=\left(x^{\prime}, x_{n}\right) \in \mathbb{R}^{n}: 0<x_{n}<h,\left|x^{\prime}\right|<a x_{n}\right\}
$$

with fixed $a>0$ such that $\partial D \subset \bigcup_{j=1}^{m} U_{j}$ and $\left(U_{j} \cap D\right)+C_{j} \subset D$ for all $j=1, \ldots, m$.

The Sobolev spaces of fractional smoothness are obtained by setting

$$
W_{p}^{s}(D):=\left\{u \in W_{p}^{\lfloor s\rfloor}(D):\|u\|_{W_{p}^{s}(D)}<\infty\right\},
$$

where

$$
\|u\|_{W_{p}^{s}(D)}^{p}:=\|u\|_{L^{p}(D)}^{p}+\sum_{|\alpha|=\lfloor s\rfloor} \int_{D \times D} \frac{\left|\partial^{\alpha} u(x)-\partial^{\alpha} u(y)\right|^{p}}{|x-y|^{n+\{s\} p}} d x d y .
$$

The defined norm $\|\cdot\|_{W_{p}^{s}(D)}$ is equivalent to the norm induced by the real method of interpolation by Remark 4.4.2/2 in [25]. Together with [25, Theorem 4.6.1(e)], we deduct the following theorem.

Theorem 2.2 Let $D \subset \mathbb{R}^{n}$ be a bounded domain of cone type, $1<p<\infty$, and $t \geq 0$. Then for all $s>t+n / p$ one has a continuous embedding $W_{p}^{s}(D) \hookrightarrow \bar{C}^{t}(D)$. The embedding is still valid for $s=t+n / p$ if $t \notin \mathbb{N}_{0}$. 
We generalize the spaces of Hölder continuous and differentiable functions $\bar{C}^{t}$ to manifolds by imposing these properties on charts. Before doing so, we recapitulate the necessary geometric definitions and properties. For more details on manifolds, we refer the reader to e.g., $[12,14,15,26]$.

If $A \subset \mathbb{R}^{n}$ is any subset, $m \in \mathbb{N}$, and $k \in \mathbb{N} \cup\{0, \infty\}$, then $f: A \rightarrow \mathbb{R}^{m}$ is $k$ times continuously differentiable or of class $C^{k}$ if for every $x \in A$ there exists an open $\mathcal{O}_{x} \subset$ $\mathbb{R}^{n}$ containing $x$ and $g: \mathcal{O}_{x} \rightarrow \mathbb{R}^{m}$ of class $C^{k}$ that coincides with $f$ on $A$. Such $f$ are collected in $C^{k}\left(A ; \mathbb{R}^{m}\right)$. Looking ahead, in order to avoid technicalities we will only consider manifolds without manifold boundary, such as the Euclidean space or a sphere therein. The first step is the definition of an atlas.

Definition 2.3 Let $M$ be a set, $r \in \mathbb{N} \cup\{0, \infty\}$, and $n \in \mathbb{N}$. A $C^{r} n$-atlas $\mathcal{A}$ on $M$ is a collection of charts $\left(U_{i}, \varphi_{i}\right), i \in I$, indexed by an arbitrary set $I$, satisfying the following:

(1) $U_{i} \subset M$ and $\bigcup_{i \in I} U_{i}=M$,

(2) $\varphi_{i}: U_{i} \rightarrow \varphi_{i}\left(U_{i}\right) \subset \mathbb{R}^{n}$ is a bijection and for any $i, j \in I, \varphi_{i}\left(U_{i} \cap U_{j}\right)$ is open in $\mathbb{R}^{n}$,

(3) $\varphi_{i} \circ \varphi_{j}^{-1}: \varphi_{j}\left(U_{i} \cap U_{j}\right) \rightarrow \varphi_{i}\left(U_{i} \cap U_{j}\right)$ is a $C^{r}$ diffeomorphism for any $i, j \in I$.

In the following, we omit the dimension $n$ in reference to an atlas. Two $C^{r}$ atlases on a set $M$ are called equivalent if their union is again a $C^{r}$ atlas on $M$. This indeed defines an equivalence relation on the $C^{r}$ atlases on $M$. The union of all atlases in such an equivalence class is again an atlas in the equivalence class, called the maximal $C^{r}$ atlas. The topology on $M$ induced by any maximal $C^{r}$ atlas is the empty set together with arbitrary unions of its chart domains.

Definition 2.4 Let $n \in \mathbb{N}$ and $r \in \mathbb{N} \cup\{0, \infty\}$. A $C^{r} n$-manifold $M$ is a set $M \neq \emptyset$ together with a maximal $C^{r} n$-atlas $\mathcal{A}(M)$ such that the induced topology is Hausdorff and paracompact.

Recall that in a Hausdorff topological space distinct points have disjoint open neighborhoods, and a topological space is called paracompact if every open cover admits a locally finite open cover (i.e., for any point, there is an open neighborhood which intersects only finitely many members of the collection) which refines the original cover. Usually, the maximal atlas is not mentioned explicitly and $M$ is understood to be equipped with the induced topology. We will say "a chart on $M$ " to refer to a chart in $\mathcal{A}(M)$. Further, we say that an atlas $\mathcal{A}$ on $M$ is an "atlas for $M$ " if it is equivalent to $\mathcal{A}(M)$. Any open subset $U \subset M$ canonically inherits the manifold structure of $M$.

Definition 2.5 Let $M$ be a $C^{r} n$-manifold and $k \geq 0$ an integer. A function $f: M \rightarrow \mathbb{R}$ is said to be of class $C^{k}$, denoted by $f \in C^{k}(M)$, if $f \circ \varphi^{-1} \in C^{k}(\varphi(U))$ for every chart $(U, \varphi)$ on $M$. The support supp $f$ of $f \in C^{0}(M)$ is the closure of the set $\{x \in M: f(x) \neq 0\}$.

For any $t \geq 0$, a function $f: M \rightarrow \mathbb{R}$ is said to be continuous (locally of class $\bar{C}^{t}$ ) if for any $x \in M$ there exists an open connected subset $V \subset M, x \in V$, such that for any chart $(U, \varphi)$ with $U \subset V$, the composite function $f \circ \varphi^{-1}: \varphi(U) \rightarrow \mathbb{R}$ is continuous(of class $\left.\bar{C}^{t}\right)$.

A useful technical device is the partition of unity defined next. 
Definition 2.6 Let $M$ be a $C^{r} n$-manifold and let $\mathcal{U}=\left\{U_{i}\right\}_{i \in I}$ be an open cover of $M$. A $C^{r}$ partition of unity subordinate to $\mathcal{U}$ is a collection $\left\{\psi_{i}\right\}_{i \in I} \subset C^{r}(M)$ such that

(1) $0 \leq \psi_{i}(x) \leq 1$ for all $i \in I$ and $x \in M$,

(2) there exists a locally finite open cover $\left\{V_{i}\right\}_{i \in I}$ of $M$ with supp $\psi_{i} \subset V_{i} \cap U_{i}$,

(3) $\sum_{i \in I} \psi_{i}(x)=1$ for all $x \in M$ (where the sum is finite by the previous assertion).

The assumed paracompactness of $M$ implies the existence of such partitions of unity, see [14, Chapter II, Corollary 3.8] or [15, Theorem 1.73], which is stated in the following proposition.

Proposition 2.7 Let $M$ be a $C^{r}$ n-manifold. Let $\mathcal{U}=\left\{U_{i}\right\}_{i \in I}$ be an open cover of $M$. Then there exists a $C^{r}$ partition of unity subordinate to $\mathcal{U}$.

We close the preparatory section by introducing random fields on manifolds. Random fields on domains are defined accordingly. In what follows, let $(\Omega, \mathcal{F}, P)$ be a probability space.

Definition 2.8 Let $M$ be a $C^{r} n$-manifold and let $\mathcal{B}(M)$ denote its Borel $\sigma$-algebra. A mapping $X: \Omega \times M \rightarrow \mathbb{R}$ that is $(\mathcal{F} \otimes \mathcal{B}(M))$-measurable is called a (real-valued) random field on the manifold $M$. A random field $Y$ is a modification of a random field $X$ if $P(X(x)=Y(x))=1$ for all $x \in M$. For any $t \geq 0$, a random field $X$ on $M$ is said to be continuous (locally of class $\bar{C}^{t}$ ) if $X(\omega)$ is continuous (locally of class $\bar{C}^{t}$ ) for all $\omega \in \Omega$.

We note that if $M$ is endowed with a metric (say, given by a Riemannian metric), and the resulting metric space is separable and locally compact, then measurability of $X(x)$ for all $x \in M$ and continuity in probability of $X$ imply $(\mathcal{F} \otimes \mathcal{B}(M))$-measurability of $X$ (cf. [20]).

\section{Hölder Continuity and Differentiability of Random Fields}

This section contains our main results on Hölder continuity and differentiability of random fields. We begin by considering random fields on domains of cone type. As indicated in the introduction, results on sample Hölder continuity on different types of domains are wellknown (see, e.g., [2, 8, 11, 21]), but sample differentiability has not been of main interest so far (see, however, $[3,22]$ for the available results). We prove sample Hölder continuity and differentiability properties in Theorem 3.1 by revisiting the approach of [8, Proof of Theorem 3.4] via the Sobolev embedding theorem. We then address sample Hölder and differentiability properties of random fields on manifolds in Theorem 3.5.

We now state our version of the Kolmogorov-Chentsov theorem on domains of cone type.

Theorem 3.1 Let $D \subset \mathbb{R}^{n}$ be a bounded domain of cone type and let $X: \Omega \times D \rightarrow \mathbb{R}$ be a random field on $D$. Assume that there exist $d \in \mathbb{N}_{0}, p>1, \epsilon \in(0, p]$, and $C>0$ such that the weak derivatives $\partial^{\alpha} X$ are in $L^{p}(\Omega \times D)$ and

$$
\mathbb{E}\left(\left|\partial^{\alpha} X(x)-\partial^{\alpha} X(y)\right|^{p}\right) \leq C|x-y|^{n+\epsilon}
$$

for all $x, y \in D$ and any multi-index $\alpha \in \mathbb{N}_{0}^{n}$ with $|\alpha| \leq d$. Then $X$ has a modification that is locally of class $\bar{C}^{t}$ for all $t<d+\min \{\epsilon / p, 1-n / p\}$. 
We remark that Eq. 1 with $\epsilon>p$ for $\alpha=0$ would imply that almost every sample of the random field is actually a constant function (cf. [4, Proposition 2]).

The proof is given in two steps. In the following lemma we first obtain a continuous modification of $X$ based on [11, Theorem 2.3.1], which we again denote by $X$. In the second step, we prove Theorem 3.1 by invoking the Sobolev embedding on $X(\omega)$ for all $\omega \in \Omega$. Since $X(\omega)$ is continuous for all $\omega \in \Omega$, this does not modify the random field, and there is no need to prove measurability of a modified field and that it is actually a modification. Alternatively, one could use the last step of [23, Proof of Theorem 2].

Let us start by showing the existence of a continuous modification.

Lemma 3.2 Under the assumptions of Theorem 3.1, X admits a continuous modification.

Proof Observe that the domain $D$ equipped with the usual Euclidean metric $|\cdot-\cdot|$ is a totally bounded pseudometric space in the sense of [11]; indeed, its metric entropy $\mathrm{D}(\delta):=$ $\mathrm{D}(\delta ; D,|\cdot-\cdot|)$ is bounded by $\mathrm{D}(\delta) \leq \tilde{C} \delta^{-n}$ for all $\delta>0$ and some constant $\tilde{C}>0$, since the domain $D$ can be embedded into a $n$-dimensional closed cube of finite diameter. We set $\Psi(r):=C r^{n+\delta}$, where the constant $C$ is provided by Eq. 1 , and $f(r):=r^{\delta / p}$ for $r \geq 0$. The integrals $\int_{0}^{1} r^{-1} f(r) d r=p / \delta$ as well as $\int_{0}^{1} \mathrm{D}(r) \Psi(2 r) f(r)^{-p} d r \leq C \tilde{C} 2^{n+\delta}$ are finite. Therefore, [11, Theorem 2.3.1] shows the existence of a continuous modification of $X$.

Having obtained a continuous modification, we are set to continue with the proof of Theorem 3.1.

Proof of Theorem 3.1 Assume without loss of generality that the random field $X$ is continuous (otherwise apply Lemma 3.2). Consider arbitrary $0<v<\min \{(n+\epsilon) / p, 1\}$ and $\alpha \in \mathbb{N}_{0}^{n}$ with $|\alpha|=d$. Since

$$
(\omega, x, y) \mapsto \frac{\left|\partial^{\alpha} X(\omega, x)-\partial^{\alpha} X(\omega, y)\right|^{p}}{|x-y|^{n+v p}}
$$

is $(\mathcal{F} \otimes \mathcal{B}(D \times D))$-measurable, we apply Fubini's theorem and hypothesis (1) to obtain

$$
\begin{aligned}
\mathbb{E}\left(\int_{D \times D} \frac{\left|\partial^{\alpha} X(x)-\partial^{\alpha} X(y)\right|^{p}}{|x-y|^{n+v p}} d x d y\right) & =\int_{D \times D} \frac{\mathbb{E}\left(\left|\partial^{\alpha} X(x)-\partial^{\alpha} X(y)\right|^{p}\right)}{|x-y|^{n+v p}} d x d y \\
& \leq C \int_{D \times D}|x-y|^{n+\epsilon-(n+v p)} d x d y .
\end{aligned}
$$

The last integral is finite due to $\epsilon-v p>-n$. With the $L^{p}(\Omega \times D)$ integrability assumptions on $X$ and its derivatives of order $d$, this implies that

$$
\mathbb{E}\left(\|X\|_{W_{p}^{d+v}(D)}^{p}\right)=\mathbb{E}\left(\|X\|_{L^{p}(D)}^{p}\right)+\sum_{|\alpha|=d} \mathbb{E}\left(\int_{D \times D} \frac{\left|\partial^{\alpha} X(x)-\partial^{\alpha} X(y)\right|^{p}}{|x-y|^{n+v p}} d x d y\right)
$$

is finite, and therefore there exists $\Omega^{\prime} \in \mathcal{F}$ with $P\left(\Omega^{\prime}\right)=1$ such that $X(\omega) \in W_{p}^{d+v}(D)$ for all $\omega \in \Omega^{\prime}$. Consider as continuous modification of $X$ the random field $\tilde{X}:=\mathbb{1}_{\Omega^{\prime}} X$, where $\mathbb{1}_{\Omega^{\prime}}$ is the indicator function of $\Omega^{\prime}$. By the Sobolev embedding Theorem 2.2, we get that $\tilde{X}(\omega) \in \bar{C}^{t}(D)$ for all $\omega \in \Omega$ and all $t<d+v-n / p$. Since $0<v<\min \{(n+\epsilon) / p, 1\}$ was arbitrary, the claim follows. 
We remark that $1-n / p$ is positive only for $p>n$. In the case that $n \geq p$, we obtain in Theorem 3.1 only lower sample differentiability order $\lfloor t\rfloor$ than the assumed weak differentiability order $d$.

Remark 3.3 The assumptions in Theorem 3.1 can be weakened. If $d \neq 0$, it is sufficient that Eq. 1 holds for $|\alpha|=d$, and that $X$ has a continuous modification as provided by Theorem 2.3.1 in [11] under the assumption that Eq. 1 holds for $\alpha=0$ and some $\epsilon>0$.

We apply Theorem 3.1 in the following example to a Brownian motion on the interval, recovering the classical property of Hölder continuity with exponent $\gamma<1 / 2$.

Example 3.4 If $X$ is a Brownian motion on the interval $[0, T] \subset \mathbb{R}^{1}, T<\infty$, then Assumption (1) is satisfied for $\alpha=0$, any $p \geq 2$, and $\epsilon=p / 2-1$. Thus $X$ admits a modification that is locally of class $\bar{C}^{t}$ for any $0<t<\sup _{p \geq 2}(p / 2-1) / p=1 / 2$, which is the well-known result.

We are now ready to generalize Theorem 3.1 to random fields on manifolds.

Theorem 3.5 Let $M$ be a $C^{r} n$-manifold, $r>0$, and let $X: \Omega \times M \rightarrow \mathbb{R}$ be a random field on $M$. Assume that there exist $d \in \mathbb{N}_{0}, p>1$, and $\epsilon \in(0, p]$ such that for any chart $(U, \varphi)$ on $M$ with bounded $\varphi(U) \subset \mathbb{R}^{n}$, there exists $C_{\varphi}>0$ such that the weak derivatives of $X_{\varphi}:=X \circ \varphi^{-1}$ satisfy $\partial^{\alpha} X_{\varphi} \in L^{p}(\Omega \times \varphi(U))$ and

$$
\mathbb{E}\left(\left|\partial^{\alpha} X_{\varphi}(x)-\partial^{\alpha} X_{\varphi}(y)\right|^{p}\right) \leq C_{\varphi}|x-y|^{n+\epsilon}
$$

for all $x, y \in \varphi(U)$ and any multi-index $\alpha \in \mathbb{N}_{0}^{n}$ with $|\alpha| \leq d$. Then $X$ has a modification that is locally of class $\bar{C}^{t}$ for all $t<d+\min \{\epsilon / p, 1-n / p\}$ with $t \leq r$.

Proof To obtain the continuous modification we first construct a locally finite atlas with coordinate domains that are bounded and of cone type. On each of these charts, a modification of $X$ is provided by Theorem 3.1. Using a partition of unity we then patch together a modification of $X$ with the desired properties.

For each $x \in M$, let $\left(\tilde{U}_{x}, \tilde{\varphi}_{x}\right)$ be a chart on $M$ with $x \in \tilde{U}_{x}$. Let $D_{x} \subset \tilde{\varphi}_{x}\left(\tilde{U}_{x}\right)$ be an open ball of positive radius centered at $\tilde{\varphi}_{x}(x)$. Define $U_{x}:=\tilde{\varphi}_{x}^{-1}\left(D_{x}\right)$ and $\varphi_{x}:=\left.\tilde{\varphi}_{x}\right|_{U_{x}}$. Let $\mathcal{A}:=\left\{\left(U_{x}, \varphi_{x}\right): x \in M\right\}$ be the resulting atlas for $M$, which we will index by $\Phi:=\{\varphi:$ $(U, \varphi) \in \mathcal{A}\}$.

Now, for each $\left(U_{\varphi}, \varphi\right) \in \mathcal{A}$, the coordinate domain $\varphi\left(U_{\varphi}\right)$ is a bounded domain with smooth boundary, in particular of cone type. With our assumptions on $X_{\varphi}$, Theorem 3.1 provides a modification $Y^{\varphi}$ of the random field $X_{\varphi}: \Omega \times \varphi\left(U_{\varphi}\right) \rightarrow \mathbb{R}$ on $\varphi\left(U_{\varphi}\right)$, which is locally of class $\bar{C}^{t}$ for any fixed $t<d+\min \{\epsilon / p, 1-n / p\}$, for each $\varphi \in \Phi$.

Let $\left\{\psi_{\varphi}\right\}_{\varphi \in \Phi}$ be a $C^{r}$ partition of unity subordinate to $\left\{U_{\varphi}\right\}_{\varphi \in \Phi}$, which exists by Proposition 2.7. Define $Y: \Omega \times M \rightarrow \mathbb{R}$ by $Y:=\sum_{\varphi \in \Phi} \psi_{\varphi} Y^{\varphi} \circ \varphi$. Since the covering $\left\{\operatorname{supp} \psi_{\varphi}\right\}_{\varphi \in \Phi}$ is locally finite, the sum is well-defined on a neighborhood of any $x \in M$. Furthermore, $Y$ is a random field on $M$ because all $\varphi \in \Phi$ are $C^{r}$ diffeomorphisms and therefore at least continuous. Moreover, it is a modification of $X$ by the properties of the partition of unity. Owing to the fact that $r \geq t$, the random field $Y$ is locally of class $\bar{C}^{t}$.

We finish this section with two comments. First, since we only used the assumptions on the random field on the charts to apply Theorem 3.1, it is clear that Remark 3.3 carries over to Theorem 3.5. Second, for an example of random fields on manifolds, we refer the reader 
to [13]. Therein, isotropic Gaussian random fields on the unit sphere in $\mathbb{R}^{3}$ are considered and sample regularity is obtained by direct calculations.

Acknowledgments The work was supported in part by ERC AdG no. 247277. The authors thank Sonja Cox, Sebastian Klein, Markus Knopf, Jürgen Potthoff, and Christoph Schwab for fruitful discussions and helpful comments, as well as René Schilling for pointing out reference [23]. The first author acknowledges the hospitality of the Seminar for Applied Mathematics during summer 2013.

\section{References}

1. Adams, R.A., Fournier, J.J.F.: Sobolev Spaces, Pure and Applied Mathematics (Amsterdam), vol. 140, 2nd edn. Elsevier/Academic Press, Amsterdam (2003)

2. Adler, J.R.: The Geometry of Random Fields. Reprint of the 1981 original ed. Society for Industrial and Applied Mathematics (SIAM), Philadelphia (2010)

3. Adler, R.J., Taylor, J.E.: Random Fields and Geometry. Springer Monographs in Mathematics. Springer, New York (2007)

4. Brézis, H.: How to recognize constant functions. A connection with Sobolev spaces. Uspekhi Mat. Nauk. 57(4(346)), 59-74 (2002)

5. Charrier, J.: Strong and weak error estimates for elliptic partial differential equations with random coefficients. SIAM J. Numer. Anal. 50(1), 216-246 (2012)

6. Chentsov, N.N.: Weak convergence of stochastic processes whose trajectories have no discontinuities of the second kind and the heuristic approach to the Kolmogorov-Smirnov tests. Theory Probab. Appl. 1(1), 140-144 (1956)

7. Cramér, H., Leadbetter, M.R.: Stationary and Related Stochastic Processes. Sample Function Properties and Their Applications. Wiley, New York (1967)

8. Da Prato, G., Zabczyk, J.: Stochastic Equations in Infinite Dimensions. Encyclopedia of Mathematics and Its Applications, vol. 44. Cambridge University Press, Cambridge (1992)

9. Evans, L.C.: Partial Differential Equations. Graduate Studies in Mathematics, vol. 19. American Mathematical Society, Providence (1998)

10. Hoffmann-Jørgensen, J.: Stochastic Processes on Polish Spaces. Åarhus Universitet, Matematisk Institut, Åarhus (1991)

11. Khoshnevisan, D.: Multiparameter Processes. An Introduction to Random Fields. Springer Monographs in Mathematics. Springer, New York (2002)

12. Klingenberg, W.P.A.: Riemannian Geometry. de Gruyter Studies in Mathematics, vol. 1, 2nd edn. Walter de Gruyter \& Co., Berlin (1995)

13. Lang, A., Schwab, C.: Isotropic Gaussian random fields on the sphere: regularity, fast simulation, and stochastic partial differential equations. SAM Report 2013-15. arXiv:1305.1170 [math.PR] (2012)

14. Lang, S.: Fundamentals of Differential Geometry, Graduate Texts in Mathematics, vol. 191. Springer, New York (1999)

15. Lee, J.M.: Manifolds and Differential Geometry, Graduate Studies in Mathematics, vol. 107. American Mathematical Society, Providence (2009)

16. Loève, M.: Probability Theory II, Graduate Texts in Mathematics, vol. 46, 4th edn. Springer, New York (1978)

17. Mittmann, K., Steinwart, I.: On the existence of continuous modifications of vector-valued random fields. Georgian Math. J. 10(2), 311-317 (2003)

18. Nousiainen, T., McFarquhar, G.M.: Light scattering by quasi-spherical ice crystals. J. Atmos. Sci. 61(18), 2229-2248 (2004)

19. Peszat, S., Zabczyk, J.: Stochastic Partial Differential Equations with Lévy Noise. An Evolution Equation Approach. Encyclopedia of Mathematics and Its Applications, vol. 113. Cambridge University Press, Cambridge (2007)

20. Potthoff, J.: Sample properties of random fields I: Separability and measurability. Comm. Stoch. Anal. 3(3), 143-153 (2009)

21. Potthoff, J.: Sample properties of random fields II: Continuity. Comm. Stoch. Anal. 3(1), 331-348 (2009)

22. Potthoff, J.: Sample properties of random fields III: Differentiability. Comm. Stoch. Anal. 4(3), 335-353 (2010)

23. Schilling, R.L.: Sobolev embedding for stochastic processes. Expo. Math. 18(3), 239-242 (2000) 
24. Slutsky, E.: Qualche proposizione relativa alla teoria delle funzioni aleatorie. Giorn. Ist. Ital. Attuari. 8, 183-199 (1937)

25. Triebel, H.: Interpolation Theory, Function Spaces, Differential Operators. North-Holland Mathematical Library, vol. 18. North-Holland Publishing Co., Amsterdam (1978)

26. Wloka, J.: Partial Differential Equations. Cambridge University Press, Cambridge. Translated from the German by C. B. Thomas and M. J. Thomas (1987) 